\title{
QUICK OBSERVATIONS OF THE FADING X-RAYS FROM GAMMA-RAY BURSTS WITH ASCA
}

T. MURAKAMI, Y. UEDA, R. FUJIMOTO, M. ISHIDA, R. SHIBATA, S. UNO, F. NAGASE AND ISAS TEAM

Institute of Space and Astronautical Science

3-1-1, Yoshinodai, Sagamihara, Kanagawa 229, Japan

A. YOSHIDA, N. KAWAI, F. TOKANI, C. OTANI AND RIKEN TEAM Institute of Physical and Chemical Research

2-1, Hirosawa, Wako, Saitama 351, Japan

AND

F.E. MARSHALL, R.H.D. CORBET, J.H. SWANK, T. TAKESHIMA, D.A. SMITH, A. LEVINE, R.A. REMILLARD, R. VANDERSPEK, (RXTE TEAM), C.R. ROBINSON, C. KOUVELIOTOU, C. MEEGAN, V. CONNAUGHTON, R.M. KIPPEN, (BATSE TEAM), K. HURLEY, (UCB), S.D. BARTHELMY (GCN), L. PIRO, E. COSTA, J. HEISE, F. FIORE, (SAX TEAM), J.V. PARADIJS, Y. TANAKA, (UOA) AND J. GREINER(AIP)

The Large International Collaborations

\section{Introduction}

Since the discovery of fading X-rays from Gamma-Ray Bursts (GRBs) with BeppoSAX (Piro et al. 1997, Costa et al. 1997), world-wide follow-up observations in optical band have achieved the fruitful results. The case of GRB 970228, there was an optical transient, coincides with the BeppoSAX position and faded (Paradijs et al. 1997, Sahu et al. 1997). These optical observations also confirmed the extended component, which was associated with the optical transient. The new transient are fading with a power-law function in time and the later observation of HST confirmed the extended emission is stable (Fruchter et al. 1997). This extended object seems to be a distant galaxy and strongly suggests to be the host.

In the case of GRB 970508, the optical transient showed the lines of absorptions which were consistent with $\mathrm{z}=0.835$, but there was no extended 
emission confirmed. The existence of the absorption and later emission at the same $\mathrm{z}$ suggest the transient at $\mathrm{z}=0.835$ but again no galaxy was confirmed yet (Djorgovski et al. 1997, Metzger et al. 1997). Based on these two cases, most are now believing that the origin of GRBs is from distant galaxies probably not from the nuclei but in the arm. However, the examples are too few to firmly convince that all GRBs come from the distant galaxies. In fact, many GRBs described below have shown no optical transient. We hope to confirm the scenario observing much local GRBs clearly associate with a galaxy. ASCA was out of this scope, because of no capability of detecting GRBs but the quick informations of SAX enabled us to monitor the fading X-rays of GRB 970228, GRB 970402 (Yoshida et al. 1997a, Feroci et al. 1997). Recently RXTE has successfully localized GRBs responding to the LOCBURST and informed their locations to us within several hours after the detections. This also enables us to observe the fading X-rays within 2 days. We have done observations of GRB 970616, GRB 970815 and GRB 970828 following the RXTE detections (Connaughton et al. 1997, Marshall et al. 1997, Smith et al. 1997, Murakami et al. 1997a, b and c). TM reviews the ASCA efforts briefly in this report.

\section{Observations}

\subsection{GRB 970228}

We have done monitor of this burst 1 week after the burst and detected the flux. The observed flux was almost our detection limit of 7.2 \pm 2.1 (1 sigma) $\times 10^{-14} \mathrm{erg} \mathrm{sec}^{-1} \mathrm{~cm}^{-2}$ in $2-10 \mathrm{keV}$. We have detected the flux but could not say anything about the variability and the spectrum. However the detected flux was fully consistent with the power-law decay in time even one week after the burst (Costa et al. 1997, Yoshida et al. 1997a).

\subsection{GRB 970402 AND GRB 970616}

We have started the monitor of GRB 970402, 2.8 days after the burst on April 5.74 (U.T.) but could not detect any flux. The $90 \%$ upper limit was about $1 \times 10^{-13} \mathrm{erg} \mathrm{sec}^{-1} \mathrm{~cm}^{-2}$ in $2-10 \mathrm{keV}$.

The observation of GRB 970616 was also done 3.5 days after the burst. At least four X-ray sources were in the SIS FOV. Two were in the reported IPN error and one was slightly outside but consistent with the IPN considering the ASCA error (Murakami et al. 1997a, Kevin et al. 1997a). One X-ray source labeled A1 in the ROSAT IAUC was very variable but fading during our observation (Greiner et al., 1997). The most probable source; A1 faded in factor of 22 between the observations of ROSAT and ASCA and A2 and A3 were clearly out of the IPN error. However A4 in the IPN error was 
not detected. Based on the informations above, we could not conclude that the A1 source was the X-ray counterpart. However, the fading during the ASCA observation strongly suggests that A1 X-ray source might be the fading X-ray counterpart of this GRB. If this were true, the fading was not simple, the X-rays faded in power-law in time in average but in short time scale, the flux was very variable. The time scale of variability was several hours. There were no optical transient for both cases.

\subsection{GRB 970815 AND GRB 970828}

We have done observation of GRB 970815, 3.2 days after the burst. Although there was an X-ray source in the FOV, which was consistent with the new IPN but not in the RXTE/ASM error (Smith et al. 1997, Kevin et al. 1997b). The intensity of this source did not show a fading and looked stable, so we rejected the possibility of an X-ray counterpart (Murakami et al. 1997b).

Soon after this burst during the IAU symposium at Kyoto, GRB 970828 was observed. We successfully reached to the source within 1.17 days after the burst. We have carried out the ASCA maneuver at the Kyoto International Hole and also from the Kyoto university through the network not from the operation room at ISAS. The precise location of the X-ray source was reported two days after the detection but there was no optical transient discovered (Murakami et al. 1997c). The detail of the ASCA observation was reported by Yoshida at the GRB workshop in Huntsville (Yoshida et al. 1997b). This was fading during our observation, so there was no doubt to be the fading X-ray counterpart but no optical counterpart discovered.

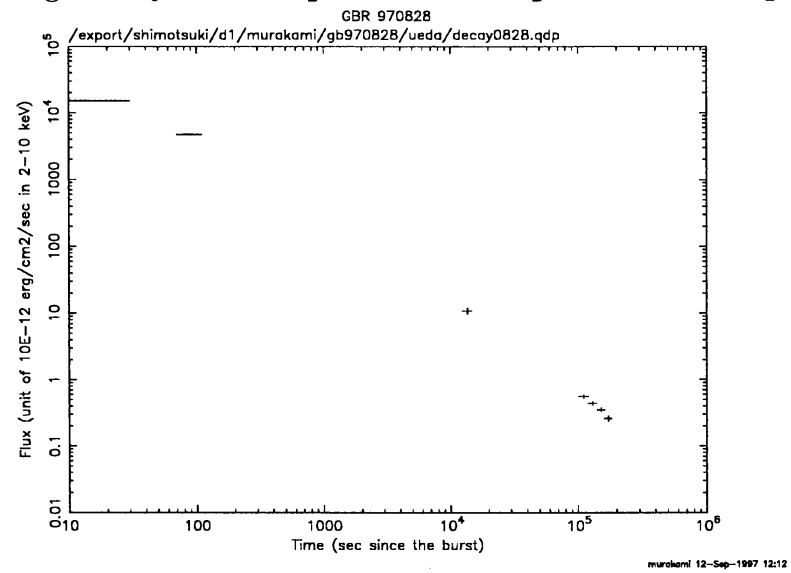

Figure 1. ASCA X-ray lightcurve of GRB 970828 together with the reported RXTE fluxes. The power-law decay index in time, between the last RXTE flux and the ASCA was about 1.44 is much faster than the case of GRB 970228 . 


\section{Discussion}

Our interest is to add another case of an optical transient and hope to find a galaxy associated with a fading X-ray counterpart. However, unfortunately there was no so far for the ASCA observations. Only the two SAX positions showed the optical transient but the four of the ASCA positions resulted no optical transient. Many ground-based observers such as Keck and Palomar have tried to find an optical transient following the ASCA notices but non. What were the differences between GRB 970228, GRB 970508 and others which were no optical transient? Let us make figure 1 of the X-ray lightcurve of GRB 970828 in the same scale of GRB 970228 (figure 3 of Costa et al. 1997). The cases without optical transient faded much faster than the cases with the optical transient. However this is still a small sampling. It is clear that the size of the bursts such as the peak flux and/or the fluency did not relate to the existence of the optical transient. What comes from this difference? We do not know the reason why. Schaefer mentioned no host galaxy is common to his deep HST observations (Schaefer et al. 1997). In any case, we require further detections of a burst which shows a slower decay and brighter flux (probably local) in future to make the origin of the optical transient clear.

Acknowledgments: This work was done by the large international collaboration. We cannot specify the contributions of each person and are afraid of missing your name. TM is responsible for that case and thanks to all the ASCA member who allowed us to carry frequent ToO observations.

\section{References}

Connaughton, V. et al., (1997) IAUC, $\mathbf{6 6 8 3}$

Costa, E. et al., (1997), Astroph, no. 9706065

Djorgovski, S. G. et al., (1997), Nature, Vol. 387, 876

Feroci, M. et al., (1997) IAUC, 6610

Fruchter, A. et al., (1997), IAUC, 6747

Greiner, J. et al., (1997), IAUC, $\mathbf{6 7 2 2}$

Hurley, K. et al., (1997a) IAUC, 6687

Hurley, K. et al., (1997b) private communication

Marshall, F. et al., (1997) IAUC, 6727

Metzger, M. R. et al., (1997), Nature, Vol. 387, 878

Murakami, T. et al., (1997a,b,c) IAUC, 6687, 6722,6729

Paradijs, van J. et al., (1997), Nature, Vol. 386, 686

Piro, L. et al., (1997), Astroph, no. 9707215

Remillard, R. et al., (1997) IAUC, 6726

Sahu, K. C. et al., (1997), Nature, Vol. 387, 476

Schaefer, B. et al., (1997), Astroph, no 9704278

Smith, D. et al., (1997), IA UC, 6718, 6728

Yoshida, A. et al., (1997) IAUC, 6593

Yoshida, A. et al., (1997) Proc. of Gamma-Ray Bursts, AIP in press at Huntsville meeting. Ed. C. Meegan. 